\title{
Hybrid Systems Verification with Isabelle/HOL: Simpler Syntax, Better Models, Faster Proofs
}

\author{
Simon Foster ${ }^{1}$, Jonathan Julián Huerta y Munive ${ }^{2}$, \\ Mario Gleirscher ${ }^{3}$, and Georg Struth ${ }^{4}$ \\ 1 University of York, UK \\ 2 University of Copenhagen, Denmark \\ 3 University of Bremen, Germany \\ ${ }^{4}$ University of Sheffield, UK
}

\begin{abstract}
We extend a semantic verification framework for hybrid systems with the Isabelle/HOL proof assistant by an algebraic model for hybrid program stores, a shallow expression model for hybrid programs and their correctness specifications, and domain-specific deductive and calculational support. The new store model yields clean separations and dynamic local views of variables, e.g. discrete/continuous, mutable/immutable, program/logical, and enhanced ways of manipulating them using combinators, projections and framing. This leads to more local inference rules, procedures and tactics for reasoning with invariant sets, certifying solutions of hybrid specifications or calculating derivatives with increased proof automation and scalability. The new expression model provides more user-friendly syntax, better control of name spaces and interfaces connecting the framework with real-world modelling languages.
\end{abstract}

\section{Introduction}

Deductive verification of hybrid systems with interactive proof assistants like Coq, HOL or Isabelle is currently gaining traction [1-8]. Such tools have reached a level of maturity, proof power, and mathematical library support that makes the development of formal methods fast, dependable and competitive. With Isabelle/HOL, an impressive theory stack for ordinary differential equations (ODEs) [9] has been combined with algebras of programs and concrete hybrid program semantics into a semantic framework for reasoning about hybrid systems [4]. Its development has so far focused on mathematical groundwork, exploring Isabelle's mathematical and theory engineering facilities, supporting verification workflows for hybrid systems, and implementing a tool prototype.

Here we report on its transformation into a formal method: (1) We supply a better hybrid program store model to reason about discrete and continuous, mutable and immutable, logical and program variables more succinctly (§3). It supports local reasoning about parts of stores through projection and framing techniques, and generation of fresh variables [10]. (2) Using this model, we derive deductive and calculational rules for hybrid system verification, including inference rules à la differential dynamic logic $(\mathrm{d} \mathcal{L})[11](\S 5)$. Rules are enhanced 
with framing conditions for local reasoning about mutable continuous variables. We supply a new ghost rule for invariant sets, a new frame rule à la separation logic, and more effective tactics, for instance for calculating framed Fréchet derivatives (§6). (3) Using Isabelle's syntax translation mechanisms we create a simpler modelling and specification language within the shallow embedding of our framework $(\S 4)$. It is meant to be extensible to modelling languages such as Modelica and computer algebra systems such as Mathematica. These contributions are explained through examples (§7); additional ones are highlighted throughout the article. Next we outline the main features of the framework to contextualise these contributions and prepare for the technical sections.

The framework [4] has been formalised as a shallow embedding using Isabelle's own language and types. The benefits of shallowness are well documented $[12,13]$. A drawback addressed by our expression model is that syntactic properties, for instance of mutable variables, may be hard to capture. The framework has three semantic layers that can be modified compositionally: abstract algebras of programs are used for deriving structural program transformation laws and verification condition generation (VCG) [14] by equational reasoning. Isabelle's type polymorphism allows their instantiation, for example with state transformer semantics and further with concrete semantics of hybrid program stores - the level at which basic commands such as assignments are modelled. The store extension to hybrid programs is obtained by a state transformer semantics for basic (continuous) evolution commands. As in $\mathrm{d} \mathcal{L}$, these commands specify ODEs (via vector fields) and guards imposing boundary conditions on their state spaces. The associated state transformer then maps initial states of evolutions to their guarded orbits, or more general sets of reachable states. Hybrid stores were modelled so far [4] as real-valued functions from program variables, using $\mathbb{R}^{n}$ with a fixed finite set of natural numbers as variable names for technical reasons. Our new hybrid store model supports more general name spaces, dynamic stores and other implementations via records or monads.

Users of the framework need to specify hybrid programs and standard correctness specifications using pre/postconditions and loop invariants. Two main workflows are then supported: (1) If ODEs have unique solutions, one can certify them or rely on automatic certifications in special cases $[8,15]$, then trigger automatic VCG and discharge the remaining verification conditions (VCs) by reasoning about solutions in state spaces. (2) More generally, one can assert invariant sets [16] for ODEs, trigger VCG and then reason abstractly about these invariants to discharge the VCs. So far, these two workflows have relied on Isabelle's internal support for equational reasoning, which seems natural for mathematicians or engineers. Our new components make them more automatic by reasoning locally with certification conditions, invariant assertions or derivatives, and more structured by supporting data-level reasoning with invariants using the derived $\mathrm{d} \mathcal{L}$-style inference rules, for those who prefer this approach. Capturing fresh variables is instrumental for our new $\mathrm{d} \mathcal{L}$-inspired ghost rule; projecting on mutable variables simplifies proof obligations and localises reasoning about such variables as does separation logic, but by different means. 
The general framework has been tested successfully on a large set of hybrid verification benchmarks [17]; our new components, integrated into an accessible simplified version of the framework, can be found online ${ }^{5}$.

\section{Semantic Preliminaries}

We first recall the basics of state and predicate transformers, the semantics of evolution commands and the set-up for our new hybrid store model $[4,18]$. The semantics can be motivated using the hybrid program syntax of $\mathrm{d} \mathcal{L}$ [11], $X::=x:=e\left|x^{\prime}=f \& G\right| ? P|X ; X| X+X \mid X^{*}$, which, beyond standard constructs of dynamic logic, features an evolution command $x^{\prime}=f \& G$. It specifies a vector field $f: T \rightarrow \mathcal{S} \rightarrow \mathcal{S}$ with time domain $T$ over a state space $\mathcal{S}$ and a guard $G: \mathcal{S} \rightarrow \mathbb{B}$, a predicate modelling boundary conditions.

State and predicate transformers. We model programs as state transformers $\alpha: \mathcal{S} \rightarrow \mathcal{P S}$, arrows of the Kleisli category of the powerset monad. (Forward) Kleisli composition $\left(\alpha \circ_{K} \beta\right) x=\bigcup\{\beta y \mid y \in \alpha x\}$ models sequential composition, the program skip is the monadic unit $\eta_{\mathcal{S}} x=\{x\}$, abort is $\lambda x . \emptyset$, nondeterministic choice $\cup$ on functions and finite iteration $\alpha^{*} x=\bigcup_{i \in \mathbb{N}} \alpha^{i} x$, with powers defined using $\circ_{K}$. Tests (and assertions) are subidentities (functions $P \leq \eta_{\mathcal{S}}$, with $\leq$ extended pointwise) mapping any $x \in \mathcal{S}$ either to $\{x\}$ or $\emptyset$. They are isomorphic to sets and predicates. Deterministic functions $\mathcal{S} \rightarrow \mathcal{S}$ are embedded into $\mathcal{S} \rightarrow \mathcal{P S}$ via $\langle\alpha\rangle=\eta_{\mathcal{S}} \circ \alpha$. Backward diamond operators (disjunctive predicate transformers with contravariant composition) are Kleisli extensions of state transformers, forward box or wlp operators are their right adjoints on the boolean algebra of tests: wlp $\alpha Q=\{x \mid \alpha x \subseteq Q\}$ for any program $\alpha$ and test $Q$. We often write partial correctness assertions $P \subseteq w / p \alpha Q$ as $\{P\} \alpha\{Q\}$.

The laws of propositional Hoare logic (ignoring assignments) are derivable in this semantics. Nevertheless, VCG with $w / p\left(\alpha \circ_{K} \beta\right)=w / p \alpha \circ$ wlp $\beta$ and $w / p$ (if $P$ then $\alpha$ else $\beta) Q=(\bar{P} \cup w / p \alpha Q) \cap(P \cap w / p \beta Q)$ is more effective, while the standard Hoare rule can be used for loops decorated with invariants.

Continuous dynamics. The evolution of continuous systems [16] is often modelled by (local) flows $\varphi: T \rightarrow \mathcal{S} \rightarrow \mathcal{S}$, where $T \subseteq \mathbb{R}$ models time and $\mathcal{S}$ a state space. Flows are assumed to be $C^{1}$-functions, and monoid actions if $T=\mathbb{R}$ : $\varphi\left(t+t^{\prime}\right)=\varphi t \circ \varphi t^{\prime}$ and $\varphi 0=i d_{\mathcal{S}}$. A trajectory $\varphi_{s}: T \rightarrow \mathcal{S}$ of $\varphi$ at $s \in \mathcal{S}$ is then a curve $\varphi_{s} t=\varphi t s$, and its orbit at $s \in \mathcal{S}$ is given by state transformer $\gamma^{\varphi}: \mathcal{S} \rightarrow \mathcal{P} \mathcal{S}, s \mapsto \mathcal{P} \varphi_{s} T$, which maps any state $s$ to the set of states on the trajectory passing through it. Flows are typically solutions to initial value problems for systems of ODEs, which specify a vector field $f: T \rightarrow \mathcal{S} \rightarrow \mathcal{S}$ assigning vectors to points in space-time, and an initial value $s \in \mathcal{S}$ at $t_{0} \in T$. A solution is then a $C^{1}$-function $X$ that satisfies $X^{\prime} t=f t(X t)$ and $X t_{0}=s$. For $f$ continuous, existence of $X$ is guaranteed by the Peano theorem. Yet $f$ must be Lipschitz continuous to guarantee uniqueness via the Picard-Lindelöf theorem,

\footnotetext{
${ }^{5}$ github.com/isabelle-utp/Hybrid-Verification, also clicking our icons.
} 
which provides intervals $U s \subseteq T$ where solutions exist around $t_{0}$ for each $s \in \mathcal{S}$ and gives rise to flows when $t_{0}=0$.

Semantics of evolution commands. Orbit maps $\gamma^{\varphi}: \mathcal{S} \rightarrow \mathcal{P S}$ are state transformers, but we generalise to include guards and to continuous vector fields. Our state transformer for evolution commands maps each state $s \in \mathcal{S}$ to the set of reachable states (or generalised orbit)

$\left(x^{\prime}=f \& G\right) s=\left\{X t \mid t \in U s \wedge\left(\forall \tau \in \downarrow_{U s} t . G(X \tau)\right) \wedge X^{\prime} t=f t(X t) \wedge X t_{0}=s\right\}$, where $\downarrow_{U s} t$ is the down-closure of $t$ in $U s$, such that $t_{0} \in U s$. It constrains the domain of existence of the solutions $X$. If we know the flow $\varphi$ for $f$, then this semantics reduces to $\left(x^{\prime}=f \& G\right) s=\left\{\varphi t s \mid t \in U s \wedge\left(\forall \tau \in \downarrow_{U s} t . G(\varphi \tau s)\right)\right\}$.

This state transformer maps any $s \in \mathcal{S}$ to all reachable states along any solution to $f$. Thus w/p $\left(x^{\prime}=f \& G\right) Q$ holds iff for every $t \in U s$, if $G(X \tau)$ holds for all $\tau \in \downarrow_{U} s$, then $Q(X t)$ holds as well. Computing wlp's within the remits of Picard-Lindelöf is then straightforward: users only need to supply and certify the flow $\varphi$ within the first workflow outlined in $\S 1$.

Invariant sets. Instead of analytic solutions, one can use the generalised orbit semantics in combination with generalised invariant sets in the second workflow. Invariant sets [16] are preserved by the orbit map of the dynamical system. With guards, more generally, $I \subseteq \mathcal{S}$ is an invariant set of $\left(x^{\prime}=f \& G\right)$ whenever $\left(x^{\prime}=f \& G\right)^{\dagger} I \subseteq I$, where $(-)^{\dagger}$ indicates Kleisli extension and yields a backward modal diamond operator. The adjunction mentioned translates this property into $I \subseteq w / p\left(x^{\prime}=f \& G\right) I$, the standard format for invariance reasoning with predicate transformers [4]. Intuitively, such invariants characterise regions of the state space that contain all orbits that respect $G$ and have a point inside them. It then suffices to assert suitable invariants in order to verify a correctness specification. We discuss invariance techniques for evolution commands in $§ 5$.

Lenses. Our algebraic model for hybrid stores is based on lenses [18, 19], a tool for manipulating program stores or state spaces, which comes in many variants and guises [20-22], in support of algebraic reasoning about program variables $[18,23]$ and local reasoning about store shapes [10].

A lens $\lambda: \mathcal{V} \Rightarrow \mathcal{S}$ is a pair $\left(\right.$ get $_{\lambda}: \mathcal{S} \rightarrow \mathcal{V}$, put $\left._{\lambda}: \mathcal{V} \rightarrow \mathcal{S} \rightarrow \mathcal{S}\right)$ such that

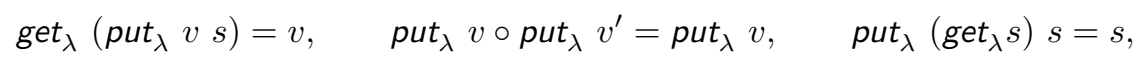

for all $v \in \mathcal{V}$ and $s \in \mathcal{S}$. Lenses are arrows in a category, as explained in $\S 3$ below. They admit many interpretations. Typically, $\mathcal{S}$ is a set of program stores. Yet $\mathcal{V}$ could be a smaller or simpler set of stores, get $s$ could forget part of $s \in \mathcal{S}$ and put $v s$ overwrite part of $s$ with $v \in \mathcal{V}$. Otherwise, $\mathcal{V}$ could be a value domain, get $s$ could look up a value $v$ in $s$ and put $v s$ could update $s$ with $v$.

We use variable lenses $x: \mathcal{V} \Rightarrow \mathcal{S}$ to model program variables $x$, get $_{x} s$ to look up values in $\mathcal{V}$ in stores $s$ and put $t_{x} v s$ to update values in $s$ by $v$. They can be implemented differently as concrete stores [23]. A variable assignment $x:=e$, with expression $e$ represented semantically as a function $\mathcal{S} \rightarrow \mathcal{V}$, is then a state transformer $(x:=e)=\left\langle\lambda s\right.$. put $\left.{ }_{x}(e s) s\right\rangle$, and $w / p(x:=e) Q=\lambda s . Q\left(\right.$ put $\left._{x}(e s) s\right)$. This suffices for VCG for hybrid programs without evolution commands. 


\section{$3 \quad$ Hybrid Store Components}

Lenses enhance our hybrid store models. They provide a unifying interface for heterogeneous variables, allow modelling frames, i.e., sets of mutable variables, and projecting parts of the global state to vector spaces of continuous evolutions. These projections are based on three lens combinators: composition, sum and quotient. This makes reasoning about hybrid stores local.

States and variables. Many modelling and programming languages support modules with local variable and constant declarations. We have implemented an Isabelle command that automates the creation of hybrid stores.

dataspace sys $=$

constants $\mathrm{c}_{1}:: \mathrm{C}_{1} \ldots \mathrm{c}_{n}:: \mathrm{C}_{n}$ assumes $\mathrm{a}_{1}: \mathrm{P}_{1} \ldots \mathrm{a}_{n}: \mathrm{P}_{n}$ variables $\mathrm{x}_{1}:: \mathrm{T}_{1} \ldots \mathrm{x}_{n}:: \mathrm{T}_{n}$

It has constants $c_{i}: C_{i}$, named constraints $a_{1}: P_{i}$ and state variables $x_{i}: T_{i}$. Inside, we can create local definitions, theorems and proofs, which are hidden, but accessible using its namespace. Internally, a locale with fixed constants and assumptions is created. Each variable is a lens $x_{i}: T_{i} \Rightarrow \mathcal{S}$, using abstract store $\mathcal{S}$ with the lens axioms as locale assumptions. We also generate independence assumptions [18] that distinguish different variables semantically. Lenses $\lambda, \lambda^{\prime}$ : $\mathcal{V} \Rightarrow \mathcal{S}$ are independent, $\lambda \bowtie \lambda^{\prime}$, if put $t_{\lambda} u \circ$ put $_{\lambda^{\prime}} v=$ put $_{\lambda^{\prime}} v \circ$ put $_{\lambda} u$, for all $u, v \in \mathcal{V}$, that is, their actions commute on all states.

Substitutions. We obtain cleaner program specifications with the notation $\sigma(x \rightsquigarrow e)=\lambda s$. put $_{x}(e s)(\sigma s)$. With this, we can describe assignments as sequences of updates: for variable lenses $x_{i}: \mathcal{V}_{i} \Rightarrow \mathcal{S}$ and "expressions" $e_{i}: \mathcal{S} \rightarrow \mathcal{V}_{i}$, $\left[x_{1} \rightsquigarrow e_{1}, x_{2} \rightsquigarrow e_{2}, \cdots\right]=i d\left(x_{1} \rightsquigarrow e_{1}\right)\left(x_{2} \rightsquigarrow e_{2}\right) \cdots$. Implicitly, any variable $y$ not mentioned in such a semantic "substitution" $\sigma: \mathcal{S} \rightarrow \mathcal{S}$ is left unchanged: $y \rightsquigarrow y$. We write e.g. $e[v / x]=e \circ[x \rightsquigarrow v]$ for the application of substitutions to expressions. These notations unclutter program specifications significantly, e.g. $(x:=e)=\langle[x \rightsquigarrow e]\rangle$ and $w / p\langle[x \rightsquigarrow e]\rangle Q=Q[e / x]$. Crucially, the Isabelle simplifier can reorder and reduce substitutions to support syntactic manipulation of variables during VCG [18]. We can extract assignments for $x$ with $\langle\sigma\rangle_{s} x=$ get $_{x} \circ \sigma$, and so e.g. $\left\langle\left[x \rightsquigarrow e_{1}, y \rightsquigarrow e_{2}\right]\right\rangle_{s} x$ reduces to $e_{1}$ when $x \bowtie y$.

Vectors and matrices. The lens category admits a forward lens composition $\lambda_{1} \stackrel{\circ}{9} \lambda_{2}: \mathcal{S}_{1} \Rightarrow \mathcal{S}_{3}$, for $\lambda_{1}: \mathcal{S}_{1} \Rightarrow \mathcal{S}_{2}, \lambda_{2}: \mathcal{S}_{2} \Rightarrow \mathcal{S}_{3}$ and units $\mathbf{1}_{\mathcal{S}}: \mathcal{S} \Rightarrow \mathcal{S}[18,22]$.

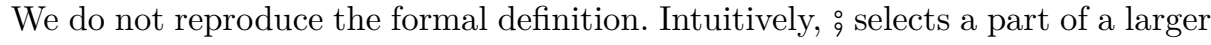
store shape, as we will shortly demonstrate.

Vectors and matrices are supported by HOL-Analysis. We supply notation $[[\mathrm{x} 11, \ldots, \mathrm{x} 1 \mathrm{n}], \ldots,[\mathrm{xm} 1, \ldots, \mathrm{xmn}]]$ for matrices and means for accessing coordinates of vectors via hybrid program variables [24]. We view vectors in $\mathbb{R}^{n}$ as part of larger hybrid stores, hence as lenses $\mathbb{R}^{n} \Rightarrow \mathcal{S}$, and project onto coordinate $v_{k}$ of any vector $\boldsymbol{v}$ in $\mathbb{R}^{n}$ using lens composition and a vector lens for $v_{k}$.

$\Pi(k:[n])=\left((\lambda s\right.$.vec-nth $s k): A^{n} \rightarrow A,(\lambda v$ s.vec-upd $\left.s k v): A \rightarrow A^{n} \rightarrow A^{n}\right)$, where $[n]=\{1 . . n\}, A^{n} \cong[n] \rightarrow A$, and the lookup function vec-nth and update function vec-upd come from HOL-Analysis. Then, for example, $v_{x}=\Pi(1) \stackrel{\bullet}{v}$ and 
$v_{y}=\Pi(2) ; \boldsymbol{v}$ for $\boldsymbol{v}: \mathbb{R}^{2} \Rightarrow \mathcal{S}$, using ; to first select the variable $\boldsymbol{v}$ and then the vector-part of the hybrid store. Obviously, $\Pi(i) \bowtie \Pi(j)$ iff $i \neq j$.

We can specify ODEs and flows via substitutions: $[\boldsymbol{p} \rightsquigarrow \boldsymbol{v}, \boldsymbol{v} \rightsquigarrow \boldsymbol{a}, \boldsymbol{a} \rightsquigarrow 0]$, e.g., specifies a vector field for lenses $\boldsymbol{p}, \boldsymbol{v}, \boldsymbol{a}: \mathbb{R}^{2} \Rightarrow \mathcal{S}$. Though ostensibly syntactic objects, these substitutions are semantically functions $\mathcal{S} \rightarrow \mathcal{S}$, and consequently can be used with Isabelle's ODE components [25,26].

Frames. Lenses support algebraic manipulations of variable frames. A frame is a set of variables that may be mutated by a program within a given context. We first show how variable sets can be modelled via lens sums. Then we define a predicate that characterises the immutable variables for programs [27]. Equipped with this we derive a frame rule in the style of separation logic that embodies local reasoning with framed variables.

Variable lenses can be combined into lenses for variable sets with lens sum [18] $\lambda_{1} \oplus \lambda_{2}: \mathcal{V}_{1} \times \mathcal{V}_{2} \Rightarrow \mathcal{S}=\left(\lambda\left(s_{1}, s_{2}\right)\right.$. $\left(\right.$ get $_{\lambda_{1}}$, get $\left._{\lambda_{2}}\right)$, put $\left.t_{\lambda_{1}} \circ p u t_{\lambda_{2}}\right)$, which is defined for $\lambda_{1}: \mathcal{V}_{1} \Rightarrow \mathcal{S}, \lambda_{2}: \mathcal{V}_{2} \Rightarrow \mathcal{S}$ if $\lambda_{1} \bowtie \lambda_{2}$. This combines two independent lenses, and can model composite variables, e.g. $(x, y)=x \oplus y$, which can be decomposed by the simplifier: $\left[(x, y) \rightsquigarrow\left(e_{1}, e_{2}\right)\right]=\left[x \rightsquigarrow e_{1}, y \rightsquigarrow e_{2}\right]$. We can also use it to specify finite sets: $\{x, y, z\}$ as $x \oplus(y \oplus z)$, yet each each variable in the sum may have a different type, e.g. $\left\{v_{x}, \boldsymbol{p}\right\}$ is a valid and well-typed construction.

Sums of lenses cannot be directly related with sets: they are only associative and commutative up-to isomorphism of cartesian products. We thus define a lens

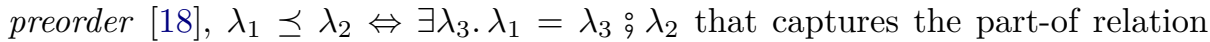
between $\lambda_{1}: \mathcal{V}_{1} \Rightarrow \mathcal{S}$ and $\lambda_{2}: \mathcal{V}_{2} \Rightarrow \mathcal{S}$, e.g. $v_{x} \leq \boldsymbol{v}$ and $\boldsymbol{p} \leq \boldsymbol{p} \oplus \boldsymbol{v}$. Lens equivalence $\cong=\preceq \cap \succeq$ then identifies lenses that have the same shape in the store. Then, for variable set lenses up-to $\cong, \oplus$ models $\cup, \bowtie$ models $\notin$, and $\preceq$ models $\subseteq$ or $\in$, which we can use to construct variable frames.

Let $A: \mathcal{V} \Rightarrow \mathcal{S}$ be a lens modelling a variable set. For $s_{1}, s_{2} \in \mathcal{S}$ let $s_{1} \approx_{A} s_{2}$ hold if $s_{1}=s_{2}$ up-to the values of variables in $A$. For $\alpha: \mathcal{S} \Rightarrow \mathcal{P S}$ define $\alpha$ nmods $A \Leftrightarrow \forall s_{1} \in \mathcal{S} s_{2} \in \alpha s_{1} \Rightarrow s_{1} \approx_{A} s_{2}$, i.e., the mutable variables in $\alpha$ are not in $A$. Then $(x:=e)$ nmods $A$ whenever $x \bowtie A$ and, recursively, $(\alpha 9 \beta)$ nmods $A$ and (if $P$ then $\alpha$ else $\beta$ ) nmods $A$ when $\alpha$ nmods $A$ and $\beta$ nmods $A$. Also, $A \leq B$ and $\alpha$ nmods $B$ implies that $\alpha$ nmods $A$.

Similarly, we use lenses to describe when a variable does not occur freely in an expression or predicate as $A \sharp e \Leftrightarrow \forall v . e\left(\right.$ put $\left._{A} v\right)=e[18]$. We can now derive a variant of separation logic's frame rule, which epitomises local reasoning:

$$
\alpha \text { nmods } A \wedge(-A) \sharp I \wedge\{P\} \alpha\{Q\} \Rightarrow\{P \wedge I\} \alpha\{Q \wedge I\}
$$

Projections. Reasoning with frames often requires localising variables to part of the store. In particular, in $\S 5$ we partition the store into continuous and discrete parts, and localise continuous variables to the former to describe their derivatives. Formally, we may use a frame lens $A: \mathcal{C} \Rightarrow \mathcal{S}$ from the global store $\mathcal{S}$ onto a local store $\mathcal{C}$. Local reasoning within $A$ requires lens quotient [10], $\lambda / / A$, which localises a lens $\lambda: T \Rightarrow \mathcal{S}$ to a lens $T \Rightarrow \mathcal{C}$. Assuming $\lambda \preceq A$, it obtains $\lambda_{1}: T \Rightarrow \mathcal{C}$ such that $\lambda=\lambda_{1} ; A$. For example, $v_{x} / / \boldsymbol{v}=\Pi(1)$ with $\mathcal{C}=\mathbb{R}^{n}$. 
Quotient cannot be defined by, e.g. $\lambda \stackrel{\circ}{B}$ for $B: \mathcal{S} \Rightarrow \mathcal{C}$, since $\mathcal{C}$ is smaller than $\mathcal{S}$, and so $B$ cannot satisfy the lens axioms.

\section{Shallow Expressions Component}

Next we present our new expression component for Isabelle that supports seamless transformations between the intuitive expression syntax often used in verification approaches and the functions $\mathcal{S} \rightarrow \mathcal{V}$ mimicking them in our shallow embedding. Naive uses of such functions may pollute specifications with $\lambda$-binders, e.g. requiring $\lambda s$. get $x s+$ get $_{y} s$ instead of $x+y$. Isabelle's syntax translations allow relating syntactic and semantic representations, and ultimately designing interfaces to modelling languages such as Modelica and Matlab.

Syntax translation. Isabelle implements a multi-stage syntax pipeline. Unicode strings are parsed and transformed into "pre-terms" [28]: elements of the ML term type containing syntactic constants. These must be mapped to previously defined semantic constants by syntax translations, before they can be checked and certified in the Isabelle kernel. Printing reverses this pipeline, mapping terms to strings. The pipeline supports a host of syntactic constructions.

We reuse this pipeline with small modifications for our bidirectional expression transformation, including pretty printing. We subject pre-terms to a lifting process, which replaces free variables and constants, and inserts store variables $(s)$ and $\lambda$-binders. Its implementation uses the syntactic annotation $(t)_{e}$ to lift the syntactic term $t$ to a semantic expression in the syntax translation rules

$$
(t)_{e} \rightleftharpoons\left[(t)^{e}\right]_{e}, \quad(x)^{e} \rightleftharpoons\left\{\begin{array}{ll}
\lambda s . g_{e t} s & \text { if } x \text { is a lens } \\
\lambda s . x & \text { otherwise }
\end{array} \quad(f t)^{e} \rightleftharpoons \lambda s . f\left((t)^{e} s\right),\right.
$$

where $p \rightleftharpoons q$ means that pre-term $p$ is translated to term $q$, and $q$ printed as $p$. Moreover, $[-]_{e}$ is a constant that marks lifted expressions that are embedded in terms. The pretty printer can then recognise a lifted term and print it.

Intuitively, $(t)_{e}$ is processed as follows. The syntax processor first parses a pre-term from string $t$. Then our parse translation traverses its syntax tree. Whenever it encounters a free variable $x$, the type system determines from the

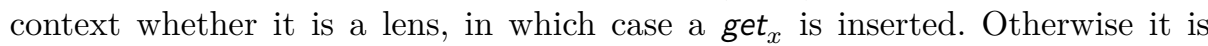
left unchanged as a logical variable. Function applications are left unchanged by $\rightleftharpoons$, except for expression constructs like $e[v / x]$. For program variables $x$ and $y$ and logical variable $z$, e.g., $\left((x+y)^{2} / z\right)_{e} \rightleftharpoons\left[\lambda s .\left(\text { get }_{x} s+\text { get }_{y} s\right)^{2} / z\right]_{e}$. Once an expression has been processed, the resulting $\lambda$-term is enclosed in $[-]_{e}$.

In assignments $x:=e$ and substitutions $[x \rightsquigarrow e], e$ is lifted transparently without user annotations. We can also lift correctness specifications and allow intuitive parsing of assertions: $\{P\} \alpha\{Q\} \rightleftharpoons(P)_{e} \leq w / p \alpha(Q)_{e}$.

Substitution. Though our expressions are functions, we can mimic syntactic substitution using the rule $(\lambda s . e(s))_{e}[v / x]=\left(\lambda s . e\left(p_{t} t_{x}(v s) s\right)\right)_{e}$. This pushes the substitution through the expression marker, by applying an update to the 
store. This rule preserves the lifted syntax, e.g. $\left((x+y)^{2} / c\right)_{e}[2 \cdot x / y]$ simplifies to $\left((3 \cdot x)^{2} / c\right)_{e}$. We can also use substitutions to determine whether a variable is used: $(x \sharp e) \Leftrightarrow \forall v .(e[v / x])=e$ : $e$ does not depend on $x$ if substituting any value $v$ for it leaves $e(\S 3)$. For example, $(5)_{e}[v / x]=(5)_{e}$, and thus $x \sharp 5$. Checking $\sharp$ is usually automatic. More generally, lenses provide enough structure for simulating many standard syntactic manipulations semantically to support VCG.

\section{Dynamical Systems Components}

We extend our components for the continuous dynamics with a notion of function framing that projects to parts of the store as outlined in §3. This supports local reasoning where evolution commands modify only continuous variables and leave discrete ones - outside the frame - unchanged. We also introduce framed differentiation to calculate differential invariants equationally, and derive a ghost rule [11] that expands ODEs with fresh variables.

Framed vector fields. We fix the continuous part $\mathcal{C}$ of hybrid store $\mathcal{S}$ with suitable topological structure for continuous variables and vector fields (e.g. $\left.\mathbb{R}^{n}\right)$ and supply a lens $\lambda: \mathcal{C} \Rightarrow \mathcal{S}=\left(\right.$ put $_{\lambda}: \mathcal{C} \rightarrow \mathcal{S} \rightarrow \mathcal{S}$, get $\left._{\lambda}: \mathcal{S} \rightarrow \mathcal{C}\right)$. We can compose this data with any $f: \mathcal{S} \rightarrow \mathcal{S}$-frame it with $\lambda$ - to $f_{\lambda}: \mathcal{S} \rightarrow \mathcal{C} \rightarrow \mathcal{C}$ such that $f_{\lambda} s=$ get $_{\lambda} \circ f \circ\left(\lambda v\right.$. put $\left.t_{\lambda} v s\right)$. For $\mathcal{C} \subseteq \mathcal{S}, f_{\lambda} s$ is thus the restriction of $f$ to $\mathcal{C}$ supplied with the full store $s$ before restriction. For example for $\mathcal{S}=\mathbb{R}^{2} \times \mathbb{R}^{2} \times \mathcal{S}^{\prime}$, $\lambda: \mathbb{R}^{2} \times \mathbb{R}^{2} \Rightarrow \mathcal{S}=(\boldsymbol{p} \oplus \boldsymbol{v})$ frames $f: \mathcal{S} \rightarrow \mathcal{S}=[(\boldsymbol{p}, \boldsymbol{v}) \rightsquigarrow(\boldsymbol{v}, 0)]$ which behaves as the identity function on $\mathcal{S}^{\prime}$, and thus $f_{\lambda} s: \mathbb{R}^{2} \times \mathbb{R}^{2} \rightarrow \mathbb{R}^{2} \times \mathbb{R}^{2}$. Similarly, if $f: T \rightarrow \mathcal{S} \rightarrow \mathcal{S}$ is a vector field and $s \in \mathcal{S}$, then $\lambda t .(f t)_{\lambda} s: T \rightarrow \mathcal{C} \rightarrow \mathcal{C}$ is the framed vector field $f_{\lambda}$ for $f$ and $s$. We can supply $f$ as a substitution $\lambda t$. [x $\left.\rightsquigarrow \boldsymbol{e} t\right]$ that describes the ODEs $\boldsymbol{x}^{\prime} t=\boldsymbol{e} t$ quite naturally, after framing.

Specifying evolution commands. We have formalised the generalised orbits in the semantics for $x^{\prime}=f \& G$ in $\S 2$ as g-orbital [4]. Here we add a framed version, g-orbital-on $\lambda f G U S t_{0}: \mathcal{S} \rightarrow \mathcal{P} \mathcal{S}$ defined as the image under $\left(\lambda v . p u t_{\lambda} v s\right)$ of $g$-orbital applied to $f_{\lambda},\left(\lambda v . G\left(\right.\right.$ put $\left.\left._{\lambda} v s\right)\right), U, S, t_{0}$, and $\left(\right.$ get $\left._{\lambda} s\right)$, where $S \subseteq \mathcal{C}$ is the codomain of $f_{\lambda}$. The application of g-orbital to $f_{\lambda}$ makes it a state transformer on $\mathcal{C}$, while its image under $\left(\lambda v\right.$. put $\left._{\lambda} v s\right)$ lifts it back to $\mathcal{S}$. VCG with the first workflow and $g$-orbital-on then remains as outlined in $\S 1$. Users need to supply flows and constants for Lipschitz continuity in order to obtain w/p's as in $\S 2$. We provide tactics that may automate this process in $\S 6$.

With Isabelle's syntax translations, we can specify g-orbital-on naturally as $\left\{x_{1}^{\prime}=e_{1}, x_{2}^{\prime}=e_{2}, \cdots, x_{n}^{\prime}=e_{n} \mid G\right.$ on $\left.U S @ t_{0}\right\}$, where each $x_{i}$ is a summand of the lens $\left\{x_{1}, \cdots, x_{n}\right\}$. We use this notation in all our examples of $\S 7$. Users can thus declare the ODEs in evolution commands coordinate-wise with lifted expressions $e_{i}$ ranging over $\mathcal{S}$, and the other parameters $G, U, S$ and $t_{0}$ which can be omitted. Their omission defaults them to constantly true, $\{t . t \geq 0\}, \mathcal{C}$ and 0 respectively. We can often collapse further to $\left(x_{1}^{\prime}, \cdots, x_{n}^{\prime}\right)=\left(e_{1}, \cdots, e_{n}\right)$ and obtain the following framing result: $\left\{\boldsymbol{x}^{\prime}=\boldsymbol{e} \mid G\right.$ on $\left.U S @ t_{0}\right\}$ nmods $(-\boldsymbol{x})$, as variables outside of $\boldsymbol{x}$ do not change during evolution and hence, by (1) in 
$\S 3$, any $I$ specified only over discrete variables is an invariant (see (12) below). We can also specify evolutions using flows with the following notation $\left\{\operatorname{EVOL}\left(x_{1}, \cdots, x_{n}\right)=\left(e_{1} \tau, \cdots, e_{n} \tau\right) \mid G\right\}$, which also carries a frame.

Framed derivatives. Isabelle supports Fréchet derivatives and we are localising them by framing. These derivatives are defined for functions between normed vector spaces or Banach spaces. Recall that, for a Fréchet differentiable function $F: \mathcal{C} \rightarrow \mathcal{T}$ at $s \in \mathcal{C}$, with $\mathcal{C} \subseteq \mathcal{S}$ open and $\mathcal{S}, \mathcal{T}$ Banach spaces, its derivative $\boldsymbol{D} F s$ is a continuous linear operator in $\mathcal{C} \rightarrow \mathcal{T}$ [29]. If $F$ is differentiable everywhere, $\boldsymbol{D} F: \mathcal{C} \rightarrow L(\mathcal{C}, \mathcal{T})$, where $L(\mathcal{C}, \mathcal{T})$ is the subspace of continuous linear operators in $\mathcal{C} \rightarrow \mathcal{T}$. For finite-dimensional spaces, $\boldsymbol{D} F s$ is the Jacobian of $F$ at $a$; compositions with unit vectors yield partial derivatives and the sum over these along the coordinates of a vector yield directional derivatives.

Fix $e: \mathcal{S} \rightarrow \mathcal{T}$ with restriction $\left.e\right|_{A} ^{s}: \mathcal{C} \rightarrow \mathcal{T}=e \circ\left(\lambda v\right.$. put $\left.{ }_{A} v s\right)$ differentiable everywhere, variable set lens $A$ and function $f: \mathcal{S} \rightarrow \mathcal{S}$. The Fréchet derivative $\mathcal{L}_{A}^{f}(e): \mathcal{S} \rightarrow \mathcal{T}$ of $e$ at $s$ framed by $A$ in direction $f$ is then defined as

$$
\mathcal{L}_{A}^{f}(e) s=\left.\boldsymbol{D} e\right|_{A} ^{s}\left(\operatorname{get}_{A} s\right)\left(\operatorname{get}_{A}(f s)\right) .
$$

Here, $\operatorname{get}_{A}(f s)$ is a vector in $\mathcal{C}$. Intuitively, in the finite dimensional case, $\left.D e\right|_{A} ^{s}\left(\operatorname{get}_{A} s\right): \mathcal{C} \rightarrow \mathcal{S}$ corresponds to a Jacobian and $\operatorname{get}_{A}(f s)$ the vector associated by $f$ to $s$ in $\mathcal{C}$ along which the directional derivative is taken. From the user perspective, after framing, $f$ supplies the vector field $f_{A}$ representing the ODEs, $s$ the values of the discrete variables, and $e$, the expression to differentiate.

Computing framed derivatives. We can calculate framed derivatives equationally. For lenses $x: V \Rightarrow \mathcal{S}, A: \mathcal{C} \Rightarrow \mathcal{S}$, function $f: \mathcal{S} \rightarrow \mathcal{S}$, and expressions $k, e_{1}, e_{2}: \mathcal{S} \rightarrow T$, which when framed by $A$ become everywhere differentiable,

$$
\begin{array}{rlrl}
\mathcal{L}_{A}^{f}(k) & =0 & \text { if } A \sharp k \\
\mathcal{L}_{A}^{f}(x) & =0 & \text { if } x \bowtie A \\
\mathcal{L}_{A}^{f}(x) & =\langle f\rangle_{s} x \quad \text { if } x \preceq A \text { and } x / / A \text { is bounded linear } \\
\mathcal{L}_{A}^{f}\left(e_{1}+e_{2}\right) & =\left(\mathcal{L}_{A}^{f}\left(e_{1}\right)\right)+\left(\mathcal{L}_{A}^{f}\left(e_{2}\right)\right) & \\
\mathcal{L}_{A}^{f}\left(e_{1} \cdot e_{2}\right) & =\left(e_{1} \cdot \mathcal{L}_{A}^{f}\left(e_{2}\right)\right)+\left(\mathcal{L}_{A}^{f}\left(e_{1}\right) \cdot e_{2}\right) & \\
\mathcal{L}_{A}^{f}\left(e^{n}\right) & =n \cdot\left(\mathcal{L}_{A}^{f}(e)\right) \cdot e^{(n-1)} & \\
\mathcal{L}_{A}^{f}(\ln (e)) & =\left(\mathcal{L}_{A}^{f}(e)\right) / e & & \text { if } e>0
\end{array}
$$

Laws (2) and (5-8) are framed analogues of known derivative rules. Laws (3) and (4) state that the derivative of a discrete variable is zero, while that for a continuous variable is extracted from $f$ as explained in $\S 3$. For the latter, we need to show that $x \preceq A$ and that $x$ localised to $\mathcal{C}$ by $A(x / / A)$ is a linear lens, that is, get $_{x / / A}$ is a continuous linear operator, implying that the expression $(x)_{e}$ is Fréchet differentiable. With these laws calculations are equational $\mathcal{L}_{x}^{[x \rightsquigarrow 1]}\left(x^{2}\right)=$ $2 \cdot\left(\mathcal{L}_{x}^{[x \rightsquigarrow 1]}(x)\right) \cdot x=2 \cdot 1 \cdot x=2 x$. Observe that changing the lens changes the part of 
$\mathcal{S}$ described by $\mathcal{C}$ and thus, the value of the framed derivative: with lens $y \bowtie x$ we get $\mathcal{L}_{y}^{[x \rightsquigarrow 1]}\left(x^{2}\right)=2 \cdot\left(\mathcal{L}_{y}^{[x \rightsquigarrow 1]}(x)\right) \cdot x=2 \cdot 0 \cdot x=0$. Also, changing the directionality changes the derivative: $\mathcal{L}_{x}^{[x \rightsquigarrow 2]}\left(x^{2}\right)=2 \cdot\left(\mathcal{L}_{x}^{[x \rightsquigarrow 2]}(x)\right) \cdot x=2 \cdot 2 \cdot x=4 x$.

Invariance checking. The fact that $I$ is an invariant for vector field $f$, as outlined in $\S 1$, has so far been modelled as diff-inv. We now supply a framed variant diff-inv-on I $\lambda f U S t_{0} G$, replacing g-orbital with g-orbital-on in the definition of invariance, where again, $\lambda: \mathcal{C} \Rightarrow \mathcal{S}$ projects onto $\mathcal{C}$ and the others parameters also remain unchanged. The adjunction between backward diamonds and wlp's still translates differential invariance to Hoare triples as in the discussion of invariant sets of $\S 2$ :

$$
\{I\}\left\{\boldsymbol{x}^{\prime}=e \mid G \text { on } U S @ t_{0}\right\}\{I\} \Leftrightarrow \operatorname{diff-inv-on~I~} \boldsymbol{x}(\lambda y .[\boldsymbol{x} \rightsquigarrow e]) U S t_{0} G \text {. }
$$

It allows us to derive the $\mathrm{d} \mathcal{L}$-style inference rules shown below. These are all proved as theorems using diff-inv-on, along with various rules for weakening and strengthening to support VCG.

$$
\begin{gathered}
\frac{G \Rightarrow\left(\mathcal{L}_{\boldsymbol{x}}^{[\boldsymbol{x} \rightsquigarrow e]} e_{1}\right) \propto^{*}\left(\mathcal{L}_{\boldsymbol{x}}^{[\boldsymbol{x} \rightsquigarrow e]} e_{2}\right)}{\left\{e_{1} \propto e_{2}\right\}\left\{\boldsymbol{x}^{\prime}=e \mid G\right\}\left\{e_{1} \propto e_{2}\right\}} \propto \in\{=, \leq,<\} \\
\frac{\left\{I_{1}\right\}\left\{\boldsymbol{x}^{\prime}=e \mid G\right\}\left\{I_{1}\right\} \quad\left\{I_{2}\right\}\left\{\boldsymbol{x}^{\prime}=e \mid G \wedge I_{1}\right\}\left\{I_{2}\right\}}{\left\{I_{1} \wedge I_{2}\right\}\left\{\boldsymbol{x}^{\prime}=e \mid G\right\}\left\{I_{1} \wedge I_{2}\right\}} \\
\frac{\boldsymbol{x} \sharp I \quad\{P\}\left\{\boldsymbol{x}^{\prime}=e \mid G\right\}\{Q\}}{\{P \wedge I\}\left\{\boldsymbol{x}^{\prime}=e \mid G\right\}\{Q \wedge I\}} \\
\frac{\left\{\bowtie \boldsymbol{x} \quad y \sharp(G, e) \quad\{I\}\left\{(\boldsymbol{x}, y)^{\prime}=(e, k \cdot y) \mid G\right\}\{I\}\right.}{\{\exists v \cdot I[v / y]\}\left\{\boldsymbol{x}^{\prime}=e \mid G\right\}\{\exists v \cdot I[v / y]\}}
\end{gathered}
$$

Rule (10) performs differential induction on (in)equalities [30]. There, $\propto^{*}$ is $\leq$ if $\propto$ is $<$, and $\propto$ otherwise: if the framed derivatives of two expressions satisfy an (in)equality, then a corresponding (in)equality is an invariant. More complex invariants (e.g. boolean combinations) reduce to these cases where computations take place [4]. If Fréchet derivatives are not defined everywhere we can still follow the procedure for invariant reasoning in [4]. Rule (11) is differential cut, framed but unchanged from [4] otherwise. It accumulates invariants in the guard sequentially during VCG. Framed differential weakening is also derived as before [4], but omitted for space reasons. Rule (12) is a frame rule that discharges invariants if they only refer to discrete variables, $\boldsymbol{x} \sharp I$.

Finally, we have also derived (13), a framed variant of the differential ghost rule of $\mathrm{d} \mathcal{L}[11]$. In $\mathrm{d} \mathcal{L}$, it substantially expands the reasoning power with invariants [31]. It is used to transform an invariant for a vector field $f$ into an invariant of $f$ extended with a fresh variable $y$ and its derivative. The extension makes the new invariant easy to prove with rules (10), (11) and (12). Formally, rule (13) says that $I$, with $y$ abstracted, is an invariant for a system of ODEs with variables in $\boldsymbol{x}$ if it is also an invariant for the same system but with $y$ as a fresh 
program variable, $\boldsymbol{x} \oplus y$, satisfying $y^{\prime}=k \cdot y$ for some constant $k$. Lens $y$ must already exists in $\mathcal{S}$, but we can always expand the store with new variables [10]. We limit the derivative of $y$ to be $k \cdot y$, but we will generalise in the future.

\section{Reasoning Components}

We have turned the results from $\S 4$ and $\S 5$ into automated proof methods for hybrid programs using the Eisbach tool [32]. These increase proof automation for both our workflows relative to [4]. Our proof methods use our baseline tactic expr-auto, which targets equalities and inequalities for shallow expressions.

hoare-wp-auto. First we supply a proof method for automatic structural VCG in our shallow approach. To discharge partial correctness specifications $\{P\} S\{Q\}$, it (1) computes w/p $S Q$ by simplification, (2) reduces substitutions and sideconditions, (3) applies expr-auto to the resulting proof goals. Our set-up ensures that the expression syntax is not exploded to HOL terms until step (3), which leads to readable data-level proofs.

dInduct. To prove goals $\{I\}\left\{\boldsymbol{x}^{\prime}=e \mid G\right\}\{I\}$, it (1) applies Law 10 to get a framed derivative expression, and (2) applies derivative calculation laws (Laws 28 ), substitution laws, and basic simplification laws. This yields derivative-free arithmetic equalities or inequalities. For cases requiring deduction, we supply dInduct-auto, which applies expr-auto after dInduct, plus further simplification lemmas from HOL-Analysis. Ultimately such heuristics should be augmented with decision procedures [33-36], as oracles or verified components.

While dInduct-auto suffices for simpler examples, differential induction must often be combined with weakening and cut rules. This leads to another more versatile proof method, using Isabelle's Eisbach [32] proof method language.

dInduct-mega. The following steps are executed iteratively until all goals are proved or no rule applies: (1) try any facts labelled with attribute facts, (2) try differential weakening to prove the goal, (3) try differential cut (Law 11) to split it into two differential invariants, (4) try dlnduct-auto. The rules are applied using some backtracking, so that if one fails, another one is tried. This automatically discharges many differential invariants, as shown in $\S 7$.

local-flow. While the proof methods so far describe the second workflow using framed versions of the inference rule of $\mathrm{d} \mathcal{L}$, the first workflow of the framework [4] supports verification with certified solutions (flows), which can be supplied using a CAS [37]. We have developed a proof method called local-flow for certifying that a flow is the unique solution to an ODE. As this involves supplying a suitable Lipschitz constant, we have written a proof method local-flow-auto that tries several such constants, such as 0.5, 1 and 2. The Picard-Lindelöf theorem can then be supplied to hoare-wp-auto and used to replace any ODE system within by its local flow. This can lead to simpler VCs than differential induction. 


\section{Examples}

Finally we evidence the benefits of our extensions to the framework [4] by examples. We illustrate the flexibility of our store model, the usability provided by shallow expressions, the local reasoning provided by frames and framed derivatives and the automation provided by our proof methods.

Circular pendulum. We begin with a small example explaining dlnduct. Consider a circular pendulum in variables $x, y: \mathbb{R} \Rightarrow \mathcal{S}$, and constant $r: \mathbb{R}$ for the radius. We use dlnduct to verify a simple invariant for its vector field.

lemma pend: " $\left\{\mathrm{r}^{2}=\mathrm{x}^{2}+\mathrm{y}^{2}\right\} \quad\left\{\mathrm{x}^{\prime}=\mathrm{y}, \mathrm{y}^{\prime}=-\mathrm{x}\right\}\left\{\mathrm{r}^{2}=\mathrm{x}^{2}+\mathrm{y}^{2}\right\} "$ by dInduct

First, the tactic yields $\mathcal{L}_{\{x, y\}}^{f}\left(r^{2}\right)=\mathcal{L}_{\{x, y\}}^{f}\left(x^{2}+y^{2}\right)$, where $f=[x \rightsquigarrow y, y \rightsquigarrow-x]$ is the vector field. It then computes $2 \cdot y \cdot x+2 \cdot(-x) \cdot y=0$ using differentiation and substitution rules, which is discharged automatically by arithmetic reasoning [4].

Water tank. Next, we formalise the classic water tank example, which requires the water level $h$ to remain between bounds $h_{l} \leq h \leq h_{u}$. A controller turns a water pump on and off to regulate $h$. The dataspace is described below.

dataspace water_tank = constants $h_{l}:: \mathbb{R} h_{u}:: \mathbb{R} c_{o}:: \mathbb{R} c_{i}:: \mathbb{R}$

assumes co:" $\mathrm{\theta}<c_{o}$ " and ci:" $c_{o}<c_{i}$ " variables flw: $: \mathbb{B} \mathrm{h}:: \mathbb{R} \mathrm{h}_{m}:: \mathbb{R}$ t: : $\mathbb{R}$

Constants $c_{o}$ and $c_{i}$ indicate rates of outflow and inflow (when the pump is on). Variable $f l w$ is for the water pump, $h_{m}$ for water level measurements, and $t$ for time. Previously, every variable had to be a real number. Now, we provide a clear separation between discrete and continuous variables; flw is of type $\mathbb{B}$.

With framed ODEs, we need only assign derivatives to variables $h$ and $t$, and the other variables are implicitly immutable during evolution:

abbreviation "dyn $\equiv \operatorname{IF}$ flw THEN $\left\{\mathrm{h}^{\prime}=-c_{o}\right.$, t' $\left.=1 \mid \mathrm{t} \leq\left(h_{l}-h_{m}\right) /\left(-c_{o}\right)\right\}$

$\operatorname{ELSE}\left\{\mathrm{h}^{\prime}=c_{i}-c_{o}, \mathrm{t}^{\prime}=1 \mid \mathrm{t} \leq\left(h_{u}-h_{0}\right) /\left(c_{i}-c_{o}\right)\right\}^{\prime \prime}$

These two ODEs model the dynamics when the inflow is on and off, respectively. They can be written intuitively due to our expression model (\$4). The frame is inferred as $\{h, t\}$, since we only assign derivatives to them, and so $h_{m}$ and flw remain unchanged during evolution, as the following theorem confirms:

lemma nm: "dyn nmods $\left\{\mathrm{fl} \mathrm{w}, \mathrm{h}_{m}\right\}$ " by (simp add: closure)

lemma $" f \mathrm{fl} \mathrm{w}=\mathrm{F}\}$ dyn $\{\mathrm{fl} \mathrm{w}=\mathrm{F}\}$ " by (rule nmods_invariant [0F nm], unrest)

Specifically, dyn modifies neither $\mathrm{flw}$ nor $h$, and so $\mathrm{flw}$ keeps its initial value $\mathrm{F}$, which is recognised as a logical variable. The latter theorem is proved using our frame rule (1). Next, we can specify our controller:

abbreviation "ctrl $\equiv\left(\mathrm{t}, \mathrm{h}_{m}\right)::=(\theta, \mathrm{h}) ;$ IF $\neg$ flw $\wedge \mathrm{h}_{m} \leq \mathrm{H}_{l}+1$ THEN flw: :=True ELSE IF flw $\wedge h_{m} \geq h_{u}-1$ THEN flw ::= False ELSE skip)"

This first assigns 0 and $h$ to $t$ and $h_{m}$, respectively, to reset the time and measure the water level. Then, if the inflow is off and the height is getting close to the minimum, then the inflow is enabled. Otherwise, if the level is getting near the maximum, then it is disabled. If neither is true, then we skip.

Using the second workflow, we use differential induction to discharge invariants for the tank dynamics. We focus on the invariant when the inflow is enabled: 


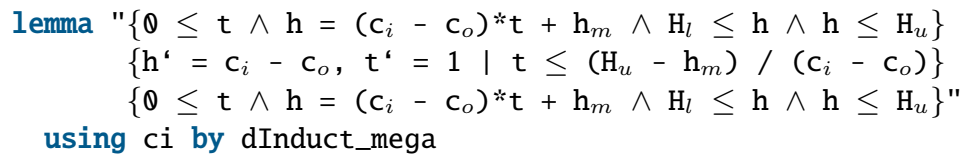

This shows one step of the verification. We prove several invariants, using $c_{i}<c_{o}$. The key is to prove that $h=\left(c_{i}-c_{o}\right) \cdot t+h_{m}$, which gives the solution for $h$ and allows us to bound how much the water rises. dlnduct-mega automates the proof with successive differential cuts. The system proof is concluded with hoare-wpauto to verify the controller. The verification is automated by a high-level proof method (dProve):

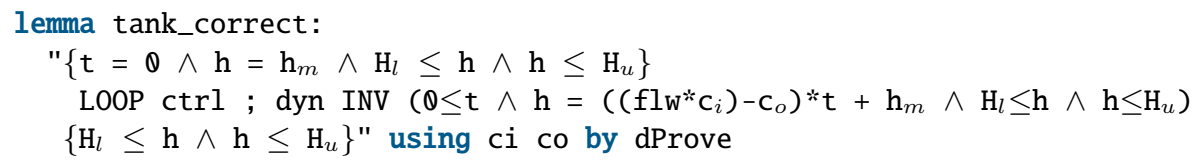

We need to supply an extended invariant for both the controller and dynamics via an annotation. Internally the proof uses the frame rule to demonstrate that both $\mathrm{flw}$ and $\neg \mathrm{flw}$ are invariants of dyn. The earlier lemma is technically not required, as dProve itself invokes dlnduct-mega during the proof.

We can alternatively verify the controller using the first workflow, with a solution to the differential equations:

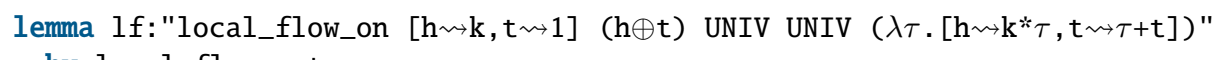
by local_flow_auto

lemma " $\left\{\mathrm{h}_{m} \leq \mathrm{h} \wedge \mathrm{h} \leq \mathrm{h}_{M}\right\}$ LOOP ctrl; dyn INV ( $\left.\mathrm{h}_{m} \leq \mathrm{h} \wedge \mathrm{h} \leq \mathrm{h}_{M}\right) \quad\left\{\mathrm{h}_{m} \leq \mathrm{h} \wedge \mathrm{h} \leq \mathrm{h}_{M}\right\}$ " using tank_arith[OF _ co ci] by (hoare_wp_auto local_flow: lf)

We need to certify the unique (framed) solution for the water tank vector field using local-flow-auto. The loop invariant need not refer to flw or $h_{m}$, since these are discrete. The proof uses hoare-wp-auto, which is given the local flow proof lf, and so can internally replace the ODE with the flow.

Exponential decay. This example contrasts the use of the differential ghost law and solutions in ODE proof. We wish to show that $x>0$ is an invariant of $x^{\prime}=-x$, which is not immediately obvious because the derivative is negative.

The proof using differential ghost is as follows: $x>0$ is equivalent to $x y^{2}=1$ for some value of $y$. To show that this new property is an invariant for the evolution command, we use the ghost rule to expand the system of ODEs into $x^{\prime}=$ $-x, y^{\prime}=y / 2$, which retains the behaviour of $x$. Then, by standard reasoning [4], $x y^{2}=1$ is an invariant because $\left(x y^{2}\right)^{\prime}=x^{\prime} y^{2}+2 x y y^{\prime}=-x y^{2}+x y^{2}=0=1^{\prime}$. The Isabelle proof requires some interaction:

lemma dG_example: " $\{\mathrm{x}>0\}\{\mathrm{x},=-\mathrm{x}\}\{\mathrm{x}>\boldsymbol{\theta}\}$ "

apply (dGhost "y" " $\left(x * y^{\wedge} 2=1\right) e^{\text {" }}$ "1/2", expr_auto add: exp_arith)

apply (dInduct_auto, simp add: power2_eq_square) done

The first line applies differential ghost, with the fresh variable $y$, the property $x y^{2}=1$, and the factor $1 / 2$ of the ODE $y^{\prime}=y / 2$. This yields $0<x \Leftrightarrow \exists v \cdot x v^{2}=$ 
1 , which we prove using a lemma (exp_arith). The last line applies dlnduct-auto and a sledgehammer [34] supplied proof for the goal $y y \neq y^{2} \Rightarrow y=0$.

For the first workflow, $x>0$ is an invariant because the solution, $x t=x_{0} e^{-t}$, is a positive exponential function for $x_{0}>0$. The proof of invariance follows immediately from this fact and automatically with our tactic.

lemma flow_ex:" $\{\mathrm{x}>\boldsymbol{\theta}\}\left\{\mathrm{x}^{\prime}=-\mathrm{x}\right\}\{\mathrm{x}>\boldsymbol{\theta}\} "$ by (hoare_wp_auto local_flow: If)

We again supply a certified solution using the theorem lf (omitted). Alternatively, if users wish to skip the certification and write the flow directly in the specification, this is also possible and automatic using notation introduced in§5.

lemma " $\{\mathrm{x}>\boldsymbol{\theta}\}\{$ EVOL $\mathrm{x}=\mathrm{x} * \exp (-\tau)\}\{\mathrm{x}>\boldsymbol{\theta}\} "$ by hoare_wp_auto

Autonomous boat. The final example [24] is a controller for an autonomous boat using the second workflow. We model the dynamics and several controller components, which demonstrates our flexible hybrid store model and local reasoning with our framework. The boat's objective is to navigate along several way-points, while avoiding obstacles. It is manoeuvrable in $\mathbb{R}^{2}$ and has a rotatable thruster generating a positive propulsive force $\boldsymbol{f}$ with maximum $f_{\max }$. The boat's state is determined by its position $\boldsymbol{p}$, velocity $\boldsymbol{v}$, and acceleration $\boldsymbol{a}$ :

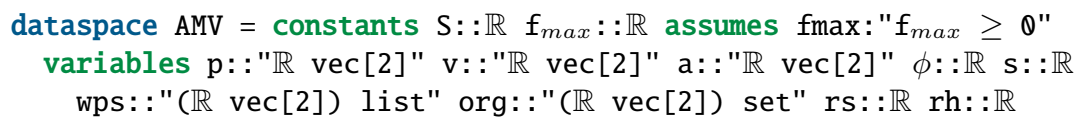

This complex store model consists of a combination of discrete and continuous variables. Here, $\mathbb{R} \operatorname{vec}[n]$ is a vector of dimension $n$. In the dataspace, we have a variable for linear speed $s$, and constant $S$ is the maximum speed. We also model discrete variables for the way-point path (wps), the obstacle register (org), and requested speed and heading (rs and rh).

With the following axiom, we relate $s, \phi$ with $\boldsymbol{v}$ and constrain $s$.

abbreviation "ax $\equiv\left(\mathrm{s}{ }_{R}[[\sin (\phi), \cos (\phi)]]=\mathrm{v} \wedge \theta \leq \mathrm{s} \wedge \mathrm{s} \leq \mathrm{S}\right)^{e "}$

This states that $\boldsymbol{v}$ is equal to $s$ multiplied by the heading unit-vector using scalar multiplication $\left({ }^{*} R\right)$ and our vector syntax, and $0 \leq s \leq S$. The kinematics $\left[\boldsymbol{p}^{\prime}, \boldsymbol{v}^{\prime}, \boldsymbol{a}^{\prime}\right]^{T}$ is specified below:

abbreviation "ODE $\equiv\left\{\mathrm{p}^{\prime}=\mathrm{v}, \mathrm{v}^{\prime}=\mathrm{a}, \mathrm{a}^{\prime}=0, \phi^{\prime}=\omega\right.$,

$$
\left.\mathrm{s}^{\prime}=\text { if } \mathrm{s} \neq \boldsymbol{\theta} \text { then }(\mathrm{v} \cdot \mathrm{a}) / \mathrm{s} \text { else }\|\mathrm{a}\| \mid \operatorname{aax}\right\}^{\prime \prime}
$$

We also specify derivatives for $\phi$ and $s$. The former, $\omega$ is the angular velocity, which has the value $\arccos ((\boldsymbol{v}+\boldsymbol{a}) \cdot \boldsymbol{v} /(\|\boldsymbol{v}+\boldsymbol{a}\| \cdot\|\boldsymbol{v}\|))$ when $\|\boldsymbol{v}\| \neq 0$ and 0 otherwise. The linear acceleration $\left(s^{\prime}\right)$ is calculated using the inner product of $\boldsymbol{v}$ and $\boldsymbol{a}$. If the current speed is 0 , then $s^{\prime}$ is simply $\|a\|$. All other variables in the store are implicitly outside the evolution frame:

lemma "ODE nmods $\{\mathrm{rs}, \mathrm{rh}$, wps, org $\}$ " by (simp add: closure) 
The controller for the AMV consists of three parts: Navigation for way-point following, AP the autopilot proportional controller, and LRE the safety controller. Navigation and LRE both supply requested headings and speeds to the Autopilot, which calculates an acceleration vector for the ODE. For reasons of space, we omit further details. We prove some differential invariants of ODE:

lemma " $\left\{\mathrm{s}^{2}=\mathrm{v} \cdot \mathrm{v}\right\}$ ODE $\left\{\mathrm{s}^{2}=\mathrm{v} \cdot \mathrm{v}\right\}$ " by (dWeaken, metis orient_vec_mag_n)

lemma " $\{\mathrm{a}=\boldsymbol{\theta} \wedge \mathrm{v}=\mathrm{V}\}$ ODE $\{\mathrm{a}=\boldsymbol{\theta} \wedge \mathrm{v}=\mathrm{V}\}$ " by (dInduct_mega)

lemma " $\{(\mathrm{a}=\boldsymbol{\theta} \wedge \mathrm{s}>\boldsymbol{\theta}) \wedge \phi=\mathrm{X}\}$ ODE $\{\phi=\mathrm{X}\}$ " by (dInduct_mega)

The first shows that $s^{2}$ is equal to the inner product of $v$ with itself, and is charged by differential weakening and a vector lemma. The second shows that if the acceleration vector is 0 , the velocity is not changing. The third shows, similarly, that the orientation is not changing. These demonstrate our ability to form differential invariants over vectors, as well as scalars. We also reason about vectors algebraically without coordinate-wise decomposition. Since $s$ and $v$ are modified only by the ODE, we can also show that the first invariant is also an invariant of each other component using our frame rule; e.g. $\left\{s^{2}=v\right\}$ Autopilot $\left\{s^{2}=v\right\}$. Moreover, this is also a system invariant. This kind of local reasoning makes proof about such component-based systems tractable. We check a final ODE invariant:

lemma $\left\{a \cdot v \geq 0 \wedge(a \cdot v)^{2}=(a \cdot a) \cdot(v \cdot v)\right\}$ ODE $\left\{a \cdot v \geq 0 \wedge(a \cdot v)^{2}=(a \cdot a) \cdot(v \cdot v)\right\} "$

by (dInduct_mega, metis inner_commute)

This property tells us that if $\boldsymbol{v}$ and $\boldsymbol{a}$ have the same direction, they will continue to do so. The boat may be linearly accelerating or decelerating, but is not turning. Method dlnduct-mega produces a proof obligation relating to inner product, which is discharged with sledgehammer [34].

\section{Related Work, Conclusions, and Future Work}

We have transformed an Isabelle framework for the verification of hybrid systems into a more user-friendly and effective formal method, using Isabelle's syntax translation mechanisms to interface with more natural modelling and specification languages for hybrid programs. We have also shown how local reasoning about hybrid stores can be achieved using lenses, how this leads to better and more automatic tactics and to more accessible verification conditions.

Deductive reasoning about hybrid systems is not new, and there is substantial work supporting this activity through domain-specific decision procedures. With PVS, a formalisation for $\mathrm{d} \mathcal{L}$-style verification by formalising semi-algebraic sets and real analytic functions is in its early steps [38]. With Coq, the ROSCoq framework [39] uses Coq's CoRN library of constructive real numbers to formalise a Logic of Events (LoE) to reason about hybrid systems. The VeriDrone project [1] uses the Coquelicot library for a variant of the temporal logic of actions. Both semantics are very different from our $\mathrm{d} \mathcal{L}$-inspired one. Yet we view the framework rather as complementary to $\mathrm{d} \mathcal{L}$ 's KeYmaera $\mathrm{X}$ tool, which brings the benefit of certified decisions procedures for real arithmetic and a user-friendly interface. With Isabelle, a term checker for $\mathrm{d} \mathcal{L}$ has been formalised as a deep embedding $[40,41]$, but without aiming at verification components. A hybrid Hoare 
logic [2] for verifying hybrid CSP processes [42] has been formalised as a shallow embedding, but with a very different semantics to our own Hoare logic [6].

Work remains to be done for transforming this framework further into an industrial-strength formal method. This is supported by its openness and compositionality. Our new hybrid store and extant ODE components for Isabelle could, for instance, be integrated into the state transformer semantics [4] with little effort. Our new expression language could easily be replaced by Modelica syntax. In the future, one could use Isabelle's code generator to provide pathways from hybrid programs to verified controller implementations. Openness implies in particular that anyone interested in hybrid systems verification with Isabelle could extend, adapt and contribute to this framework. Its power is only limited by Isabelle's higher order logic and the mathematical and verification components that the community provides for it.

\section{Acknowledgements}

This work is funded by UKRI-EPSRC project CyPhyAssure (grant reference EP/S001190/1); Novo Nordisk Fonden Start Package Grant (NNF20OC0063462); and the Assuring Autonomy International Programme (AAIP; grant CSI:Cobot), a partnership between Lloyd's Register Foundation and the University of York.

\section{References}

1. Ricketts, D., Malecha, G., Alvarez, M.M., Gowda, V., Lerner, S.: Towards verification of hybrid systems in a foundational proof assistant. In: MEMOCODE, IEEE (2015) 248-257

2. Wang, S., Zhan, N., Zou, L.: An improved HHL prover: An interactive theorem prover for hybrid systems. In: ICFEM. Volume 9407 of LNCS. (2015) 382-399

3. Huerta y Munive, J.J., Struth, G.: Verifying hybrid systems with modal Kleene algebra. In: RAMiCS. Volume 11194 of LNCS., Springer (2018) 225-243

4. Huerta y Munive, J.J., Struth, G.: Predicate transformer semantics for hybrid systems: Verification components for Isabelle/HOL. arXiv:1909.05618 [cs.LO] (2019)

5. Foster, S.: Hybrid relations in Isabelle/UTP. In: UTP. Volume 11885 of LNCS., Springer (2019) 130-153

6. Foster, S., Huerta y Munive, J.J., Struth, G.: Differential Hoare logics and refinement calculi for hybrid systems with Isabelle/HOL. In: RAMiCS[postponed]. Volume 12062 of LNCS. (2020) 169-186

7. Huerta y Munive, J.J.: Verification components for hybrid systems. Archive of Formal Proofs (2019)

8. Huerta y Munive, J.J.: Matrices for ODEs. Archive of Formal Proofs (2020)

9. Immler, F., Traut, C.: The flow of ODEs: Formalization of variational equation and Poincaré map. J. Automated Reasoning 62(2) (2019) 215-236

10. Foster, S., Baxter, J.: Automated algebraic reasoning for collections and local variables with lenses. In: RAMiCS. Volume 12062 of LNCS., Springer (2020)

11. Platzer, A.: Logical Foundations of Cyber-Physical Systems. Springer (2018) 
12. Boulton, R.J., Gordon, A.D., Gordon, M.J.C., Harrison, J., Herbert, J., Tassel, J.V.: Experience with embedding hardware description languages in HOL. In: IFIP Transactions. Volume A-10., North-Holland (1992) 129-156

13. Wildmoser, M., Nipkow, T.: Certifying machine code safety: Shallow versus deep embedding. In: TPHOLs. Volume 3223 of LNCS., Springer (2004) 305-320

14. Nipkow, T., Klein, G.: Concrete Semantics - With Isabelle/HOL. Springer (2014)

15. Huerta y Munive, J.J.: Affine systems of ODEs in Isabelle/HOL for hybrid-program verification. In: SEFM. Volume 12310 of LNCS., Springer (2020) 77-92

16. Teschl, G.: Ordinary Differential Equations and Dynamical Systems. AMS (2012)

17. Mitsch, S., Huerta y Munive, J.J., Jin, X., Zhan, B., Wang, S., Zhan, N.: ARCHCOMP20 category report: Hybrid systems theorem proving. In: ARCH20. (2019) $141-161$

18. Foster, S., Baxter, J., Cavalcanti, A., Woodcock, J., Zeyda, F.: Unifying semantic foundations for automated verification tools in Isabelle/UTP. Science of Computer Programming 197 (October 2020)

19. Foster, S., Zeyda, F.: Optics. Archive of Formal Proofs (May 2017)

20. Oles, F.: A Category-Theoretic Approach to the Semantics of Programming Languages. PhD thesis, Syracuse University (1982)

21. Back, R., von Wright, J.: Refinement Calculus-A Systematic Introduction. Springer (1998)

22. Foster, J.: Bidirectional programming languages. PhD thesis, University of Pennsylvania (2009)

23. Foster, S., Zeyda, F., Woodcock, J.: Unifying heterogeneous state-spaces with lenses. In: ICTAC. Volume 9965 of LNCS. (2016) 295-314

24. Foster, S., Gleirscher, M., Calinescu, R.: Towards deductive verification of control algorithms for autonomous marine vehicles. In: ICECCS, IEEE (October 2020)

25. Immler, F., Hölzl, J.: Numerical analysis of ordinary differential equations in Isabelle/HOL. In: ITP. Volume 7406 of LNCS., Springer (2012) 377-392

26. Hölzl, J., Immler, F., Huffman, B.: Type classes and filters for mathematical analysis in Isabelle/HOL. In: ITP. Volume 7998 of LNCS., Springer (2013) 279294

27. Foster, S., Nemouchi, Y., Gleirscher, M., Wei, R., Kelly, T.: Integration of formal proof into unified assurance cases with Isabelle/SACM. Formal Aspects of Computing (2021)

28. Kuncar, O., Popescu, A.: A consistent foundation for Isabelle/HOL. J. Autom. Reasoning 62 (2019) 531-555

29. Cheney, E.W.: Analysis for applied mathematics. Springer (2001)

30. Platzer, A.: The structure of differential invariants and differential cut elimination. Logical Methods in Computer Science 8(4) (2008)

31. Platzer, A., Tan, Y.K.: Differential equation axiomatization: The impressive power of differential ghosts. In: LICS, ACM (2018) 819-828

32. Matichuk, D., Murray, T.C., Wenzel, M.: Eisbach: A proof method language for Isabelle. J. Automated Reasoning 56(3) (2016) 261-282

33. Hölzl, J.: Proving inequalities over reals with computation in Isabelle/HOL. In: PLMMS, ACM (2009) 38-45

34. Blanchette, J.C., Kaliszyk, C., Paulson, L.C., Urban, J.: Hammering towards QED. Journal of Formalized Reasoning 9(1) (2016)

35. Li, W., Passmore, G., Paulson, L.: Deciding univariate polynomial problems using untrusted certificates in Isabelle/HOL. J. Autom. Reasoning 62 (2019) 29-91

36. Cordwell, K., Yong, K.T., A., P.: A verified decision procedure for univariate real arithmetic with the BKR algorithm. In: ITP. (2021) To appear. 
37. Hickman, T., Laursen, C.P., Foster, S.: Certifying differential equation solutions from computer algebra systems in Isabelle/HOL. arXiv:2102.02679 [cs.LO] (February 2021)

38. Slagel, J.T., White, L., Dutle, A.: Formal verification of semi-algebraic sets and real analytic functions. In: CPP, ACM (2021) 278-290

39. Anand, A., Knepper, R.A.: Roscoq: Robots powered by constructive reals. In: ITP. Volume 9236 of LNCS., Springer (2015) 34-50

40. Bohrer, B., Rahli, V., Vukotic, I., Völp, M., Platzer, A.: Formally verified differential dynamic logic. In: CPP, ACM (2017) 208-221

41. Platzer, A.: Differential game logic. Archive of Formal Proofs 2019 (2019)

42. Liu, J., Lv, J., Quan, Z., Zhan, N., Zhao, H., Zhou, C., Zou, L.: A calculus for hybrid CSP. In: APLAS. Volume 6461 of LNCS., Springer (2010) 1-15 